\title{
SeisSol on Distributed Multi-GPU Systems: CUDA Code Generation for the Modal Discontinuous Galerkin Method
}

\author{
Ravil Dorozhinskii \\ Technical University of Munich \\ Garching bei München, Germany \\ ravil.dorozhinskii@tum.de
}

\author{
Michael Bader \\ Technical University of Munich \\ Garching bei München, Germany \\ bader@in.tum.de
}

\begin{abstract}
We present a GPU implementation of the high order Discontinuous Galerkin (DG) scheme in SeisSol, a software package for simulating seismic waves and earthquake dynamics. Our particular focus is on providing a performance portable solution for heterogeneous distributed multi-GPU systems. We therefore redesigned SeisSol's code generation cascade for GPU programming models. This includes CUDA source code generation for the performance-critical small batched matrix multiplications kernels. The parallelisation extends the existing MPI+X scheme and supports SeisSol's cluster-wise Local Time Stepping (LTS) algorithm for ADER time integration.

We performed a Roofline model analysis to ensure that the generated batched matrix operations achieve the performance limits posed by the memory-bandwidth roofline. Our results also demonstrate that the generated GPU kernels outperform the corresponding cuBLAS subroutines by 2.5 times on average. We present strong and weak scaling studies of our implementation on the Marconi100 supercomputer (with 4 Nvidia Volta V100 GPUs per node) on up to 256 GPUs, which revealed good parallel performance and efficiency in case of time integration using global time stepping. However, we show that directly mapping the LTS method from CPUs to distributed GPU environments results in lower hardware utilization. Nevertheless, due to the algorithmic advantages of local time stepping, the method still reduces time-to-solution by a factor of 1.3 on average in contrast to the GTS scheme.
\end{abstract}

\section{CCS CONCEPTS}

- Applied computing $\rightarrow$ Earth and atmospheric sciences.

\section{KEYWORDS}

Discontinuous Galerkin, ADER, GPU, SeisSol, seismic wave propagation, high performance computing, code generation, local time stepping

\section{ACM Reference Format:}

Ravil Dorozhinskii and Michael Bader. 2021. SeisSol on Distributed MultiGPU Systems: CUDA Code Generation for the Modal Discontinuous Galerkin Method . In The International Conference on High Performance Computing in Asia-Pacific Region (HPCAsia 2021), January 20-22, 2021, Virtual Event,

Permission to make digital or hard copies of all or part of this work for personal or classroom use is granted without fee provided that copies are not made or distributed for profit or commercial advantage and that copies bear this notice and the full citation on the first page. Copyrights for components of this work owned by others than ACM must be honored. Abstracting with credit is permitted. To copy otherwise, or republish, to post on servers or to redistribute to lists, requires prior specific permission and/or a fee. Request permissions from permissions@acm.org.

HPCAsia 2021, January 20-22, 2021, Virtual Event, Republic of Korea

(C) 2021 Association for Computing Machinery.

ACM ISBN 978-1-4503-8842-9/21/01 . .\$15.00

https://doi.org/10.1145/3432261.3436753
Republic of Korea. ACM, New York, NY, USA, 14 pages. https://doi.org/10. $1145 / 3432261.3436753$

\section{INTRODUCTION}

SeisSol ${ }^{1}$, is a software package for simulating seismic waves and earthquake dynamics. As many applications, it has been originally designed and tuned for traditional multicore and manycore x86 CPU platforms [11]. To achieve high performance while keeping the solver extensible for new physical models based on the modal Discontinuous Galerkin (DG) method [25], SeisSol follows a software design based on code generation through the open source library YATeTo $^{2}$ [26], thus achieving up to $50-60 \%$ of the peak CPU performance [26, 27], Most of the performance critical parts of SeisSol are generated at compile time using parameters provided by the user. The DG discretisation scheme, in particular, is expressed via element-local chains of matrix and tensor operations, for which YATeTo can perform additional algorithmic optimization steps that a usual compiler cannot. YATeTo generates sequences of small and dense matrix multiplications [11, 26, e.g.], which for best-possible performance on CPUs may be executed by respective hardware-optimised libraries. For x 86 architectures, SeisSol uses the LIBXSMM library [12], which again relies on code generation and can exploit knowledge that is only available at runtime, especially regarding the size of the matrices. The resulting code generation cascade opens opportunities to optimize SeisSol for different architectures. However, as it evolved from a CPU-oriented performance engineering process, it also posed substantial challenges to port and optimize SeisSol for GPU (Graphics Processing Unit) architectures.

In this paper, we present the first step towards a performanceportable version of SeisSol for GPUs: a GPU-optimized version of SeisSol's elastic wave propagation solver. This task was not trivial for two reasons: (a) SeisSol's software design does not permit us to map CPU code directly to GPU code mainly due to the code generation design, and (b) the GPU programming model itself is more restrictive than the $\mathrm{CPU}$ one, which forced us to reconsider task decomposition. Furthermore, we were constrained to find a solution that allows us to preserve the high performance of SeisSol on CPU-based systems as well as to provide decent performance on distributed multi-GPU systems without drastic changes in the code base and supporting as many SeisSol specific features as possible.

We first (in Section 2) discuss related work, pointing out that SeisSol's DG variant leads to a very particular performance characteristic. In Section 3, we give an overview of its DG scheme, including aspects of realising a cluster-based local time stepping

\footnotetext{
${ }^{1}$ www.seissol.org; software version on https://github.com/SeisSol/SeisSol.git, SHA b1b45751 was used in this paper.

${ }^{2}$ https://github.com/SeisSol/yateto.git, SHA 3f5d5481 was used in this paper.
} 
scheme in SeisSol. Section 4 explains the HPC concepts followed by SeisSol, in particular the code generation approaches and the parallelisation concepts, which are important to understand the design decisions for the GPU parallelisation. Based on this, Section 5 presents the main innovations that were necessary for a high-performance GPU parallelisation: (1) changing the task decomposition used for parallelisation from a parallel loop over elements towards a parallelisation over batched BLAS operations, (2) changing the software structure and implementation of SeisSol and YATeTo to support both CPU and GPU parallelism and (3) providing hardware-optimised code for the batched matrix multiplications. For the latter, we embedded an additional code generation phase, which is decoupled from both SeisSol and YATeTo, and is provided by a dedicated library for generating small, batched matrix multiplication CUDA kernels: GemmForge ${ }^{3}$. GemmForge exploits state-of-the-art techniques to optimise batched multiplications of matrices of small size (known at compile time) and thus achieve better performance than general-purpose batched-BLAS libaries.

In Section 6, we analyse the performance achieved by GPU code generation using a Roofline model for a model batched-BLAS setup and using SeisSol's performance proxy application. We show that we achieve substantial performance gains due to the GemmForge code generation, but we also identify performance bottlenecks for small problem sizes that become relevant for cluster-based local time stepping. Finally, in Section 7, we demonstrate the performance and scalability on a widely used benchmark for seismic wave propagation on the Marconi100 supercomputer, which features four Nvidia Volta V100 GPUs per compute node.

\section{COMPARISON TO RELATED WORK}

High-order Finite-Element and DG methods generally cope well with implementation on GPU-accelerated computing environments, such that quite a lot of previous work exists, see for example [1, 18, $20,21,23,28]$. In the following, we therefore restrict ourselves to specifics of our approach and how it relates to other work.

In contrast to most other DG approaches [1,21, 28, e.g.], our research is based on the modal DG method and therefore leads to different considerations of block/grid GPU-thread distributions. In particular, modal DG has bigger but less nested loops than for example spectral-element-oriented DG (DG-SEM). Moreover, SeisSol adopts the ADER time integration scheme (see Section 3), which entails different algorithmic characteristic and arithmetic intensity. In particular, the performance-critical space-time predictor of ADER-DG does not occur in Runge-Kutta or similar multi-stage schemes that require several grid traversals per time step.

Local time stepping (LTS), i.e., adapting the time step size to individual elements, is crucial to limit the time to solution for relevant problem settings that require approximation of complicated geophysical setups (subduction zones, e.g. [27]). Rietmann et al. [21] demonstrated impacts of local time stepping on strong scaling of SPECFEM3D's wave propagation solver on GPU distributed memory systems. We identify similar load balancing challenges arising from LTS and overheads caused by small time clusters. We therefore add a performance analysis for LTS both w.r.t. number of floating point operations per second, which provides further insight

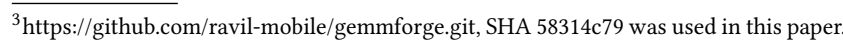

of issues related to scalability of the LTS method on distributed multi-GPU systems.

Vincent et al. [28] also used code generation for their GPU implementation of the DG method. However, their approach seems to follow rather a template-oriented code generation approach, which differs from the code generation for general tensor-operations, as provided by YATeTo in SeisSol [26]. YATeTo offers a domain-specific language with several distinct compiling stages: lexical, semantical, optimization and code generation. However, performance concept and design decisions of YATeTo are CPU-"centric", as it has been designed and tuned with traditional multi- and many-core CPU systems in mind. How to adapt the respective software design to GPU computing platforms as well as to reach portable performance on CPU- and GPU-based supercomputers, is therefore a key contribution of this paper.

\section{NUMERICAL SCHEME}

We consider elastic wave propagation, which is governed by Hooke's law and the dynamic relationship between accelerations and stresses within an elastic medium. The resulting model can be written as a linear hyperbolic PDE system in first-order formulation:

$$
\frac{\partial Q}{\partial t}+A(\mathbf{x}) \frac{\partial Q}{\partial x}+B(\mathbf{x}) \frac{\partial Q}{\partial y}+C(\mathbf{x}) \frac{\partial Q}{\partial z}=S
$$

where $Q$ is a vector of unknowns, namely: stresses and particle velocities, $Q=\left(\sigma_{x x}, \sigma_{y y}, \sigma_{z z}, \sigma_{x y}, \sigma_{y z}, \sigma_{x z}, u, v, w\right)$ at a point $\mathbf{x}=$ $(x, y, z) \in \mathbb{R}^{3}$ and time $t \in \mathbb{R} ; A(\mathbf{x}), B(\mathbf{x})$ and $C(\mathbf{x})$ are the spacedependent Jacobian matrices; $S$ is the external source term which, as $Q$, in general depends on $\mathbf{x}$ and $t$.

SeisSol employs a high-order discontinuous Galerkin (DG) method on unstructured adaptive tetrahedral meshes. Using a set of purely space-dependent, orthogonal polynomial basis functions $\Phi(\xi)$ up to some degree $N$, where $\xi=(\xi, \eta, \zeta)$ are coordinates within the reference element, a numerical solution of a quantity $Q_{p}$ at any point of the reference element can be written as:

$$
Q_{p}=\sum_{l=1}^{\mathcal{B}} Q_{l p} \cdot \Phi_{l}(\xi)
$$

where $p$ is an index of a quantity. $\mathcal{B}$ is the number of basis functions given as $\mathcal{B}=\frac{1}{6} O(O+1)(O+2)$, where $O$ is the specified spatial approximation order. $Q_{l p}$ are the degrees of freedom (DOF). To simplify notation, we denote $Q_{l p}$ as $Q_{k}$ in the context when we are talking about DOF of a particular element $k$, and as $Q_{k(i)}$ when we are referencing to DOF of an $i$ th neighbor element of $k$.

For time integration, SeisSol adopts the Arbitrary high-order DERivatives (ADER) scheme, where the main components are a Taylor expansion in time and the Cauchy-Kovalewski (CK) procedure. A detailed explanation and derivation of the ADER-DG method can be found in [8] and [7]. The method allows to build a high order predictor-corrector scheme. Integrating a Taylor expansion of DOF within a time interval $\left[t^{n}, t^{n+1}\right]$ allows to compute time-predicted values of DOF $\mathcal{D}_{k}$ as follows:

$$
\mathcal{D}_{k}=\sum_{j=0}^{O-1} \frac{\left(t^{n+1}-t^{n}\right)^{j+1}}{(j+1) !} \frac{\partial^{j}}{\partial t^{j}} Q_{k}^{n}
$$


The CK procedure substitutes time derivatives with discretized space derivatives. Applying a recursive scheme and starting from $\frac{\partial^{0}}{\partial t^{0}} Q_{k}^{n}=Q_{k}^{n}$, we can evaluate time derivatives as follows:

$$
\begin{aligned}
\frac{\partial^{j+1}}{\partial t^{j+1}} Q_{k}^{n}=M^{-1}\left[\left(K^{\xi}\right)^{T}\left(\frac{\partial^{j}}{\partial t^{j}} Q_{k}^{n}\right) A_{k}^{*}\right. & +\left(K^{\eta}\right)^{T}\left(\frac{\partial^{j}}{\partial t^{j}} Q_{k}^{n}\right) B_{k}^{*} \\
& \left.+\left(K^{\zeta}\right)^{T}\left(\frac{\partial^{j}}{\partial t^{j}} Q_{k}^{n}\right) C_{k}^{*}\right]
\end{aligned}
$$

where $M$ is the mass matrix, and $K^{\xi}, K^{\eta}$ and $K^{\zeta}$ are stiffness matrices of the reference element. $A_{k}^{*}, B_{k}^{*}$ and $C_{k}^{*}$ are linear combinations of space-dependent Jacobian matrices, which result from a transformation to the reference element space. Note that equation (4) does not contain a contribution from the right-hand side because of the choice of the external source term described in 3.1.

Using the approximation (2) and the ADER-DG method applied to (1), an explicit updating scheme can be schematically written as:

$$
\begin{aligned}
Q_{k}^{n+1, *} & =Q_{k}^{n}-I_{\text {surf }}^{\text {local }}\left(\mathcal{D}_{k}\right)+I_{\mathrm{vol}}\left(\mathcal{D}_{k}\right)+I_{\text {src }} \\
Q_{k}^{n+1} & =Q_{k}^{n+1, *}-I_{\text {surf }}^{\text {nghb }}\left(\mathcal{D}_{k(i)}\right)
\end{aligned}
$$

where $Q_{k}^{n+1}$ and $Q_{k}^{n}$ are DOFs at time steps $n+1$ and $n$ respectively; $I_{\text {surf }}^{\text {local }}, I_{\text {surf }}^{\text {nghb }}, I_{\text {vol }}$ and $I_{\text {src }}$ are the main computational macro-kernels - i.e., local surface, neighbor surface, volume and external source integrals.

For completeness, we present the main macro-kernels involved in the update scheme, which can be derived using the DG machinery:

$$
\begin{aligned}
I_{\mathrm{vol}}\left(\mathcal{D}_{k}\right) & =M^{-1}\left(K^{\xi} \mathcal{D}_{k} A_{k}^{*}+K^{\eta} \mathcal{D}_{k} B_{k}^{*}+K^{\zeta} \mathcal{D}_{k} C_{k}^{*}\right) \\
I_{\text {surf }}^{\text {local }}\left(\mathcal{D}_{k}\right) & =\frac{1}{|J|} M^{-1}\left(\sum_{i=1}^{4}\left|S_{i}\right| F^{-, i} \mathcal{D}_{k} \widehat{A}_{k}^{+}\right) \\
I_{\text {surf }}^{\text {local }}\left(\mathcal{D}_{k(i)}\right) & =\frac{1}{|J|} M^{-1}\left(\sum_{i=1}^{4}\left|S_{i}\right| F^{+, i, j_{k}, h_{k}} \mathcal{D}_{k(i)} \widehat{A}_{k(i)}^{-}\right)
\end{aligned}
$$

where $\left|S_{i}\right|$ is the area of the $i$ th face; $|J|$ is the determinant of the Jacobian matrix resulting from the transformation from physical coordinates to coordinates of the reference element; $F^{-, i}$ and $F^{+, i, j_{k}, h_{k}}$ are flux matrices over the $i$ th face of the reference element, where $j_{k}$ and $h_{k}$ are indices that determine the relative position of the $k$ th element with respect to its neighbor $k(i)$ which depends on a mesh generator [7]; $\widehat{A}_{k}^{+}$and $\widehat{A}_{k(i)}^{-}$are two flux solvers of the face-local Riemann problems.

\subsection{Modeling External Sources}

SeisSol can model earthquake rupture processes using point sources in the form of

$$
S_{p}=s_{p}(t) \cdot \delta\left(\mathbf{x}-\mathbf{x}_{\mathbf{s}}\right),
$$

which is suitable for many geophysical applications. $s_{p}(t)$ is a source time function of a component $p ; \delta$ is the Dirac Delta distribution and $\mathbf{x}_{\mathbf{s}}$ the location of a source.

Applying the DG machinery to (10), using one-dimensional Gaussian integration with respect to time and properties of the Dirac Delta distribution, we obtain

$$
I_{\mathrm{src}}=M^{-1}\left(\Phi_{l}\left(\xi_{s}\right) \cdot \sum_{j=1}^{n_{G}} \omega_{j} \cdot s_{p}\left(\tau_{j}\right)\right)
$$

where $\xi_{s}=\left(\xi_{s}, \eta_{s}, \zeta_{s}\right)$ are the coordinates of a source inside the reference element; $n_{G}$ is the number of Gaussian points; $\omega_{j}$ are integration weights and $\tau_{j}$ are the corresponding time locations of the quadrature rule over time interval $\left[t^{n}, t^{n+1}\right]$. The term $I_{\text {src }}$ contributes to (5) only if a source is located inside of an element.

Following [16], SeisSol uses components of the rotated seismicmoment tensor $\widehat{M}(t)$ as source time functions $s_{p}(t)$. This allows to evaluate the time functions using only material and geometrical properties of a fault plane as well as the fault slip rate $s(t)$, which are given as an input to a simulation.

\subsection{Local Time Stepping}

As an explicit time stepping scheme, ADER is stable under the element-wise Courant-Friedrichs-Lewy (CFL) condition, which is determined by the local wave speed and the element size and shape. A computational mesh can contain grid cells that enforce much smaller time steps than in the bulk of a domain. This can be due to adaptive mesh refinement, for example at fault planes, but also due to badly shaped elements generated when matching the grid to complex 3D fault geometries (e.g., where subduction faults meet the surface at very small angles). Therefore SeisSol uses cluster-wise Local Time Stepping (LTS) to speed up the time integration process. In this section we will describe the main ideas of the method briefly. See [2,27] for a detailed discussion of the algorithmic details and of the possible speed-ups of the method on CPU-based supercomputers.

The method assigns mesh elements to different time clusters based on their individual allowed time step sizes. Clusters are updated in a multi-rate fashion (with factor 2 or 3 difference in time step size between clusters) with a cluster-wide uniform time step. Compared to global time stepping (GTS), which is always restricted by $\Delta t_{\text {min }}$ of the "smallest" mesh element, LTS updates clusters with "big" elements much less frequently while preserving the CFL condition for clusters containing small elements. Depending on the scenarios (cf. [2, 27], e.g.), the LTS scheme can achieve algorithmic speedups of $4 \times$ or more over GTS at the cost of a slightly reduced parallel efficiency due to the algorithmic complexity. Fig. 1 shows a representative distribution of elements over 7 distinct time clusters with adjacent update cluster ratio $R=2$. In addition, Fig. $12 \mathrm{~b}$ shows an example of LTS clustering depicted along a mesh cross-section where each color represents a distinct LTS cluster.

SeisSol allows the user to set arbitrary (integer) adjacent cluster update ratios. In this work, we stuck to $R=2$, the smallest possible, which leads to the maximum possible number of clusters.

\section{HPC CONCEPTS IN SEISSOL}

\subsection{Code Generation}

In SeisSol, the numerical scheme discussed in Section 3 is expressed via tensor notation i.e., considering matrices as two dimensional tensors. This generalization allows SeisSol to be easily extended to new models - e.g., modeling wave propagation phenomena with viscoelastic attenuation [15] or for fused ensemble simulations (cmp. [3]) - using the same apparatus and performance considerations when some terms become $n$-dimensional tensors. It is important to stress that the sizes and sparsity patterns of all tensors involved in the method are known a priori, as they are based on 


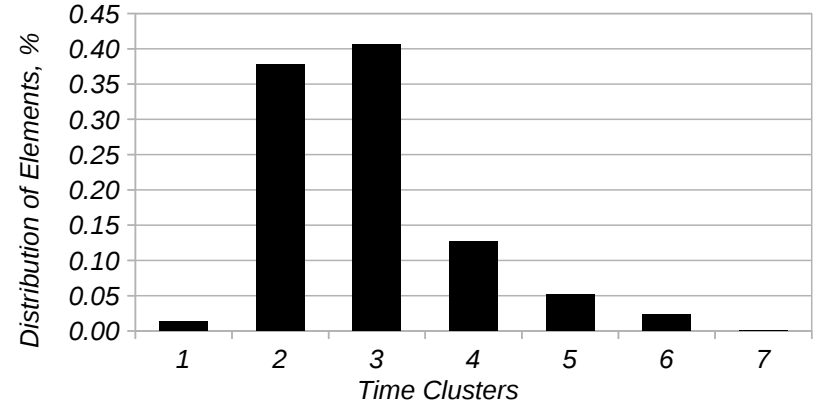

Figure 1: Example of distribution of elements over time clusters (created for the LOH.1 benchmark scenario, see Section 7.1). $R$ equal 2 means cluster $i$ is updated twice as frequently as $i+1$.

the number of physical quantities, the specific convergence order $O$ and the choice of polynomial basis functions $\Phi_{l}(\xi)$.

The efficiency of the ADER-DG method in SeisSol mainly depends on an efficient implementation of a tensor contraction algorithm which is usually imposed by two constraints at the same time, namely: maximization of hardware performance and minimization of time-to-solution. It is quite challenging to find a generic solution for this problem. However, knowledge of dimensionalities and sparsity patterns of tensors at compile time allows one to apply many optimization techniques in advance to achieve high performance.

In SeisSol, this problem is addressed by a dedicated code generator, YATeTo [26]. Tensor expressions, required for a particular physical model, are written in YATeTo's domain specific language, from which efficient $\mathrm{C}++$ code is generated. The generation phase is performed automatically while compiling SeisSol (see Fig. 2a). The generated code, compiled together with the SeisSol source files, is called from SeisSol at run-time. Fig. $2 b$ illustrates how the scope of the code generation changes from element-wise computations for CPUs (solid lines) to batched computations for GPUs (dashed lines, see detailed discussion in Section 5.5).

YATeTo splits a tensor expression into sequences of binary tensor operations and builds an Abstract Syntax Tree (AST). The optimization first traverses a resultant AST to calculate an EQuivalent SParsity Pattern (EQSPP) for each tensor. The EQSPP algorithm computes a new sparse tensor shape which includes only elements that will not get zeroed out during run-time computations. As a result, the method eliminates unnecessary calculations. The library then traverses the AST another time to generate a concrete sequence of binary tensor operations. An optimal sequence, which YATeTo aims to determine, leads to a reduced number of floating point operations. The outcome of both optimization stages is supposed to reduce time-to-solution.

YATeTo implements a binary tensor contraction using Loopover-GEMM (LoG), as described in [6]. The procedure slices tensors into a series of two dimensional sub-tensors and contracts them via a General Matrix-Matrix Multiplication (GEMM) subroutine. In general, LoG is not the most efficient technique, according to [22], however, it results in very good hardware performance especially for the small tensor sizes which we obtain in modeling the wave propagation phenomena with the ADER-DG method. Here, tensors can fully fit into the top-most caches.

To summarize, SeisSol's concept for achieving high performance is to use code generation by YATeTo to break down all tensor operations of the DG implementation into sequences of small matrix multiplications. To execute these, SeisSol relies on heavily hardwareoptimized libraries, in particular LIBXSMM [12].

\subsection{MPI+X model in SeisSol}

SeisSol follows the hybrid MPI+X model, which allows us to decouple inter- and intra-node levels of parallelism. In theory, this makes it possible to easily change one part leaving the other intact. The intra-node parallelism in SeisSol on CPU-based platforms is implemented with OpenMP. The MPI+OpenMP combination allows SeisSol to scale up to thousands of compute-nodes without a significant loss of performance [2,27]. In this sub-section, we briefly explain the main aspects of each part.

4.2.1 MPI parallelism. For distributed-memory parallelization, we partition the computational domain between multiple computenodes. In general, distributing 3D unstructured meshes of problems subjected to the LTS algorithm is not straightforward and difficult concerning load balance. SeisSol employs ParMETIS [14] for parallel partitioning, using a heuristically derived definition of work load $w$, which is assigned to each mesh element (node):

$$
w_{k}=R^{L-l_{k}}
$$

where $L$ is the total number of clusters and $l_{k}$ is a linear index of the time cluster to which element $k$ belongs. Time clusters are sequentially ordered based on their update rates, starting from the highest one. For edges, we use uniform weights.

This heuristic leads to a partitioning where each MPI process is assigned several (but not necessary all) well connected time clusters to operate on. Each partition is augmented with ghost and copy layers for exchanging information with other processes. Asynchronous MPI calls are inserted between implementations of equations (3) and (5) as well as (5) and (6) - for details see [2].

4.2.2 OpenMP parallelism. SeisSol contains a time step manager that schedules local time clusters and updates dependencies between them. Given a cluster, SeisSol distributes its elements between all available CPU cores using the parallel-for OpenMP directive for both (5) and (6). Each core loops over its assigned elements and updates DOFs. Each partial element update (Eq. (5) and (6)) can be viewed as a CPU task.

4.2.3 SIMD parallelism. SeisSol delegates all issues related to singlecore CPU performance to YATeTo. Based on a target CPU architecture and available application libraries, YATeTo either generates GEMMs using LIBXSMM [12], or inserts GEMM calls to BLAS(-like) libraries e.g., Intel MKL, OpenBLAS or Eigen. This design allows YATeTo to deliver vectorized tensor expressions. In case of a very sparse matrix multiplication or absence of the aforementioned libraries, YATeTo generates GEMM code by itself either by unrolling of a sparse matrix multiplication or by using multiple nested forloops. High single-core CPU performance is thus mainly achieved by intra-instruction parallelism and abundant data caching. 


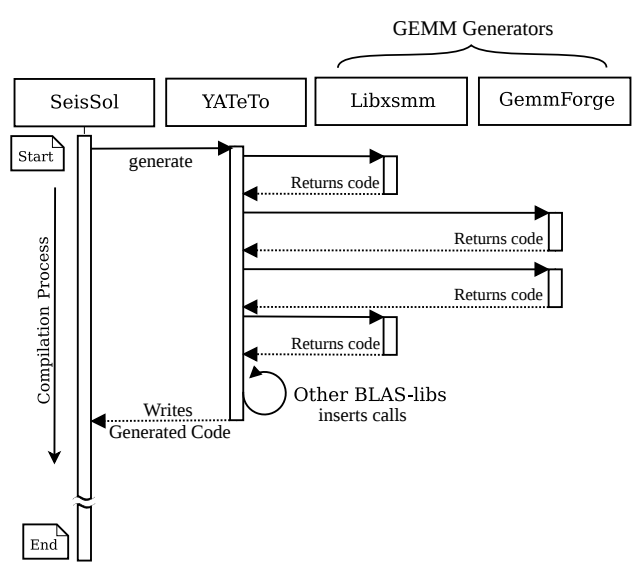

(a) Code generation process during compilation.

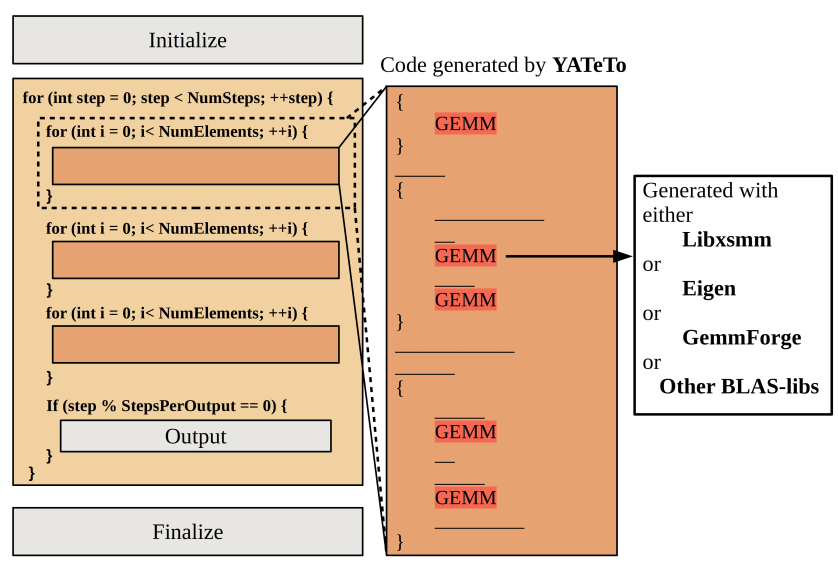

(b) Simplified source code structure.

Figure 2: Schematic description of the overall SeisSol software architecture.

\section{IMPLEMENTATION}

To profit from the flexibility of the MPI+X model and CUDA-aware implementations of the MPI standard, the design of our GPU solver is based on substituting the X part with the CUDA programming model instead of OpenMP for CPU platforms. This approach allowed us to retain key parts of SeisSol, such as mesh partitioning, LTS cluster scheduling, etc. While the original MPI+OpenMP model may assign a single MPI process per compute-node, CPU or NUMA domain, our GPU solver requires us to have as many processes as GPUs. Thus each GPU instance is controlled by a single MPI rank even in case of operating on a shared memory environment.

In the following, we discuss changes required to adapt SeisSol to the GPU programming model from the perspective of a single MPI process.

\subsection{Task Decomposition for GPU Parallelism}

An element-wise implementation of the ADER-DG scheme written as a single GPU kernel (similar to the original CPU implementation, see 4.2.2) would result in a giant GPU kernel scope containing various (sometimes nested) conditional branches e.g., caused by types of boundary conditions, relative positions of neighboring reference elements, etc. It would require to implement many SeisSol and YATeTo specific data management $\mathrm{C}++$ classes in the CUDA C language, which is much more restrictive than conventional CPU high-level languages. Moreover, finding correct CUDA block/grid parameters would become nontrivial or it would result in excessive use of CUDA dynamic parallelism. Additionally, this solution would require drastic code changes in both SeisSol and YATeTo, and most probably would not result in high performance. In short, this approach, which results in high performance on CPU platforms, contradicts to the offloading nature of GPU programming. Therefore, we had to change the original task decomposition (see 4.2.2) to adapt the GPU programming model.

A task represented as an element-wise execution of a tensor expression seems to be a rational solution. It can be viewed as moving the outer loop, which iterates over all elements of a time cluster, down to each tensor expression generated by YATeTo (cmp. Fig. 2b, dashed vs. solid scope). It requires to extend YATeTo to generate batched, instead of element-wise, source code. This step leads to two major advantages. Firstly, it avoids branching at run-time, because data required for each execution path (tensor expression) can be recorded in advance (see 5.2) and passed to the generated code as arrays of pointers. Secondly, it leads to high arithmetic intensity per kernel execution, if the implementation utilizes intelligent data caching to the shared memory and prefetching. However, some expressions involve lengthy chains of tensor contractions and additions, for example (9), and would therefore risk in low performance due to a lack of run-time GPU resources i.e., registers, shared memory, etc. This could be avoided by splitting a kernel into several ones. However, it would require a good algorithm or heuristic that is able to precisely estimate the GPU resources, especially registers, during the code generation phase. Nevertheless, the issue related to the block/grid GPU threads distribution for maximizing GPU occupancy for each kernel is still nontrivial to solve.

Thus, in this work, we perform the task decomposition at the level of binary tensor operations to avoid the aforementioned issues. It can be viewed as moving the outer loop one further level down to each tensor operation. We tolerate that this decomposition might not be optimal, e.g., due to a lower arithmetic intensity, and defer respective optimizations to future work. As for elastic wave propagation all involved tensors are of rank 2 (i.e., matrices), we focus on batched GEMM computations. However, research into extending our implementation to handle general tensor contractions is already in progress.

\subsection{Source Code Changes in SeisSol}

To be able to move the main parallel loop down to the level of batched GEMM operations, SeisSol needs to pass arrays of pointers (in the following referred to as batches) to the code generated by YATeTo for all operands involved in a tensor expression. In contrast to the original implementation where individual pointers to data, scattered within the main memory space, can be easily retrieved on the fly using pointer-based arithmetic, batches need to get recorded in advance to avoid substantial run-time overheads. 
Table 1: Matrix sizes for typical approximation orders.

\begin{tabular}{|l|c|c|}
\hline \multirow{2}{*}{ Matrices } & \multicolumn{2}{|c|}{ Order } \\
\cline { 2 - 3 } & $\mathbf{6}$ & $\mathbf{7}$ \\
\hline$Q_{k}^{n}, \mathcal{D}_{k}$ & $56 \times 9$ & $84 \times 9$ \\
\hline$M, K^{\xi}, K^{\eta}, K^{\zeta}$ & $56 \times 56$ & $84 \times 84$ \\
\hline$A_{k}^{*}, B_{k}^{*}, C_{k}^{*}$ & $9 \times 9$ & $9 \times 9$ \\
\hline$F^{-, i}, F^{+, i, j_{k}, h_{k}}$ & $56 \times 56$ & $84 \times 84$ \\
\hline$\widehat{A}_{k}^{+}, \widehat{A}_{k(i)}^{-}$ & $9 \times 9$ & $9 \times 9$ \\
\hline
\end{tabular}

We therefore introduced a new pre-processing phase that records batches for each tensor equation inside of each time cluster. The batches to operands are stored in a 2-level hash table. The outer table allows to retrieve an inner table that stores batches of all operands involved in a concrete tensor expression. A key of the outer table is a C-structure that contains four binary encoded fields: a macro-kernel name, a kernel type, a local face ID of the reference element and a face relation with the corresponding face of a neighbor element. The first field is always compulsory. The rest can be omitted if they are redundant for a particular case. Binary encoding allows to exploit the logical $A N D$ operation to embed conditional statements inside of the keys of the outer table. Sometimes this becomes necessary due to multiple possible execution paths e.g., caused by the presence of multiple boundary conditions. Keys of an inner table are integer encoded names of operands. Because SeisSol uses a static adaptive mesh, all elements of the 2-level hash table can be recorded only once: right before the beginning of a simulation.

At first glance, accessing such a data structure may seem to be a time-consuming operation. However, taking the asynchronous kernel execution nature of the CUDA programming model into account, a data search is often overlapped with kernel execution.

\subsection{GPU data management}

Our GPU implementation of SeisSol is based on the assumption that all data required for computations of the elastic wave propagation solver fit into GPU global memory. This assumption relies on experience from previous work on optimizing SeisSol for Intel's Xeon Phi co-processor [11, esp.], where this assumption has already proven to hold for the typical production scenarios. Therefore, we allocate and initialize the respective data on GPUs during the last initialization steps of SeisSol. Further data transfer of the wave propagation data is then only necessary for I/O purposes, such that respective overheads are easily amortized in typical production simulations. Data required to evaluate point sources are scattered within the entire memory space because of their arbitrary locations within a computational domain. Therefore, we mostly utilize unified memory, which results in implicit copying data back and forth within the main computational loop between CPUs and GPUs based on the page-fault mechanism of an Nvidia driver. As the data volume required for point sources is typically quite small, the overheads due to these implicit data transfers are small. Even for the case when more extensive earthquake sources are modelled by a series of point sources, these point sources typically reside close to each other, which increases probability that a transferred page will contain data for several sources at once.

\subsection{Changes in YATeTo}

We introduced three major changes in YATeTo: Firstly, we extended YATeTo to generate the source code for both CPU and GPU architectures at the same time. This allowed us to move computationally intensive parts of SeisSol to GPU while leaving non-trivial tasks on CPU e.g, data initialization, computations of point sources, etc. Secondly, we changed the interfaces between SeisSol and the generated code to accept additional parameters for GPU computing: arrays of pointers instead of regular pointers; the number of elements in a batch; additional GPU memory, allocated in advance and controlled as a stack, for holding temporary results of binary matrix operations. Lastly, we substituted regular GEMM calls with the batched ones in code generated for GPU computing.

\subsection{Generating Batched GEMM Operations}

In general, a batched GEMM operation can be expressed as:

$$
C_{e}=\alpha \cdot O p\left(A_{d}\right) \cdot O p\left(B_{f}\right)+\beta \cdot C_{e}
$$

where $C \in \mathbb{R}^{m \times n} ; A \in \mathbb{R}^{m \times k} ; C \in \mathbb{R}^{k \times n} ; e$ is a batch operation index; $d$ is either batch operation index $e$ or is omitted if $A$ is singletons; $f$ is the same as $d$ but with respect to matrix $B ; O p(X)$ can be either $X$ or $X^{T}$. Because SeisSol inherited the Fortran data layout, we only consider matrix operations in the column-major format.

Nvidia hardware comes with a collection of optimized HPC libraries and algorithms. CUTLASS, MAGMA and cuBLAS are extended implementations of the BLAS library designed for Nvidia GPU architectures. They support batched computations and tend to deliver performance close to the maximum (or peak). However, the libraries do not usually operate optimally with extremely small matrices. A custom solution can significantly outperform the libraries, if it utilizes specific information about the operands known at compile time $[9,17$, e.g.]. Table 1 shows the information known to us a priori for the elastic wave propagation model expressed with the ADER-DG scheme and for convergence orders 6 and 7.

We developed a new python library, GemmForge, callable from YATeTo during the code generation phase. The library generates a batched matrix multiplication kernel given descriptions of each operand, namely: the number of rows and columns; the total shape that contains information about how rows and columns are padded; an addressing mode; and a boolean value indicating whether an operand needs to get transposed.

The addressing mode allows the user to pass a batched operand into a kernel in three different ways: as an array of pointers, as a base pointer to strided matrices or as a pointer to a single matrix. This provides a more flexible interface to a kernel in contrast to libraries like cuBLAS, where the addressing must be the same for all involved operands. Besides generating a GPU kernel, the library generates a corresponding launcher which performs task decomposition by computing parameters of a block and a grid based on estimated required run-time resources and a number of batched operations.

Inspired by [4], our implementation performs a single matrix multiplication as a sum of outer products computed in parallel by up to 2 or 3 consecutive warps, called as a team. The team size, $m_{a}$, is equal to the number of rows of the first operand aligned to the warp size 32 . Because of the column-major matrix storage, a load or a store of any column of $A$ and $C$ by the first $m$ team threads, 


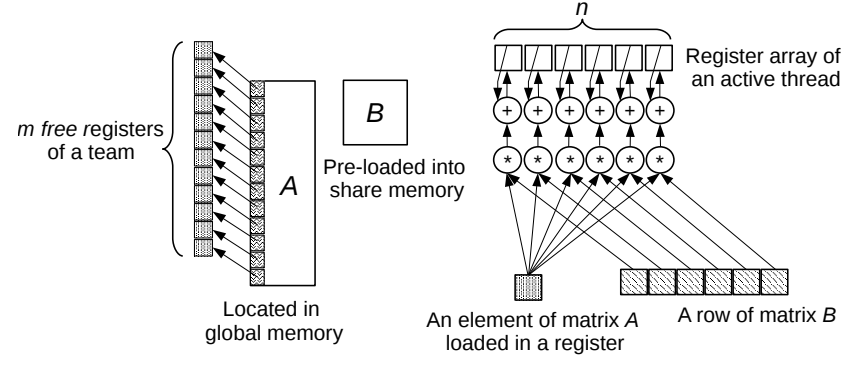

Figure 3: One iteration of a matrix multiplication kernel implemented as parallel outer product. Left: Coalesced memory read-access. Right: Work of a single active thread.

called as active threads, leads to coalesced memory access. If matrix $A$ is transposed it is pre-loaded into the shared memory, transposed on the fly and padded with zeros to avoid shared memory bank conflicts. Each active thread allocates $n$ registers, initialized with zeros, as an array to hold intermediate results of a matrix product. In each iteration, an active thread loads its corresponding element of a column of $A$ from the global GPU memory into another free register, and computes $n$ partial updates using elements of matrix $B$, copied into the shared memory at the beginning of the kernel execution. After $k$ steps, the matrix product is completely computed but distributed row-wise between arrays of active threads. $n$ additional steps are required to move the data from the register file into the global memory. The store operations are accompanied by multiplications by $\alpha$ and $\beta$ on the fly. The process is illustrated in Fig. 3. A code listing of a generated kernel is provided in Appendix A.

All active threads simultaneously load the same elements of matrix $B$ during a single kernel iteration and, therefore, make readaccess to the same banks of the shared memory. Therefore, all read operations of matrix $B$ result in broadcasts rather than completely serialized access due to bank conflicts.

An important advantage of the code generation approach is that it allows us to propagate all constants known at compile time directly to the corresponding kernels and launchers. For example, a register-based array allocation, discussed above, is only possible because the array size is known at compile time. Otherwise, the array would reside in local memory and memory accesses to the array elements would have the same high latency as global memory accesses. As another example, propagated constants allow us to efficiently apply loop unrolling, which reduces the number of control-flow operations, and provide hints to the nvcc compiler for better register allocation and utilization e.g., launch bound.

To increase the number of resident threads per Streaming Multiprocessor (SM), we allow a block to contain several teams to perform multiple matrix operations. Therefore, a block can be twodimensional if there are enough run-time resources. We estimate the availability of these based on the length of the register array required for an active thread, the amount of the required shared memory and hardware limits.

In general, a combination of the algorithm, constants propagation, compiler hints and predictions of optimal thread/block distributions at compile time results in high performance of batched

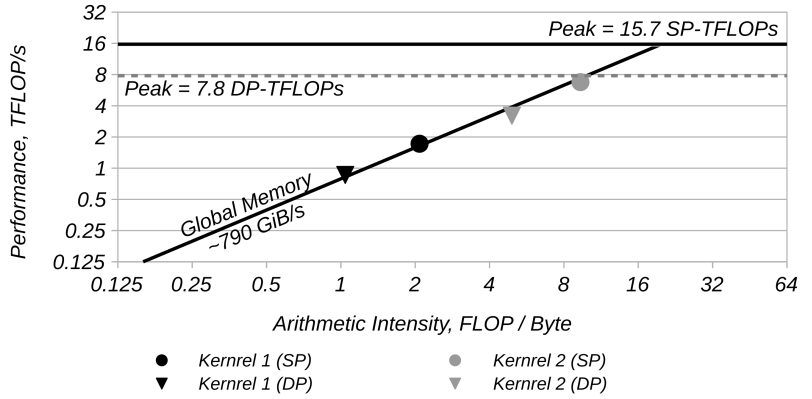

Figure 4: Correlation of obtained results with the Roofline model outline.

GEMM operations for matrix sizes specific for SeisSol, as it will be shown in the subsequent section.

\section{BENCHMARKING}

Having analyzed all steps of the integration scheme, discussed in Sec. 3, we designed a benchmark in the following form:

$$
L_{e}=D \cdot A_{e} \cdot B_{e}+L_{e}
$$

where $L, A \in \mathbb{R}^{\mathcal{B} \times 9}$ and $B \in \mathbb{R}^{9 \times 9}$, where 9 stands for the number of physical quantities. $D \in \mathbb{R}^{\mathcal{B} \times \mathcal{B}}$ represents either a mass or stiffness matrix and is therefore unique for the entire batch. The concrete implementation of (14) consists of two GEMM kernels and therefore needs some temporary storage $T \in \mathbb{R}^{\mathcal{B} \times 9}$ :

$$
\begin{array}{r}
T_{e}=A_{e} \cdot B_{e} \\
L_{e}=D \cdot T_{e}+L_{e}
\end{array}
$$

In this section, we use a single Nvidia Tesla V100 graphics card to collect performance metrics and perform comparisons. Results of this and subsequent sections are obtained using both double and single precision floating points formats (DP and SP).

\subsection{Roofline Model Analysis}

Fig. 4 shows a Roofline model that was obtained by collecting effective hardware characteristics using a benchmark similar to one described in [13] and the officially documented peak performance from [19]. We ran the GEMM kernels with a batch size equal to approximately 250000 elements and $\mathcal{B}$ equal to 56 , which correlates to the convergence order 6 . All experiments were repeated 20 times. The mean values are placed in the Roofline model in Fig. 4.

We estimate the arithmetic intensities of both kernels, $I_{k 1}$ and $I_{k 2}$, with respect to data transfers to and from the global memory. For simplicity, it is enough to consider a single batched operation in both cases. For the second kernel, we take into account that matrix $D$ is the same for all elements in a batch and, therefore, stays in L1 cache during the entire kernel execution:

$$
I_{k 1}=\frac{2 \cdot \mathcal{B} \cdot 9 \cdot 9}{(2 \cdot \mathcal{B} \cdot 9+9 \cdot 9) \cdot b} \text { and } I_{k 2}=\frac{2 \cdot \mathcal{B} \cdot 9 \cdot \mathcal{B}+\mathcal{B} \cdot 9}{(3 \cdot \mathcal{B} \cdot 9) \cdot b}
$$

where $b \in(4,8)$ is the number of bytes required to represent a floating point number (factors 9 reflect the number of quantities).

The results shown in Fig. 4 justify our estimates of the computational intensities and the assumption that matrix $D$ is cached. 


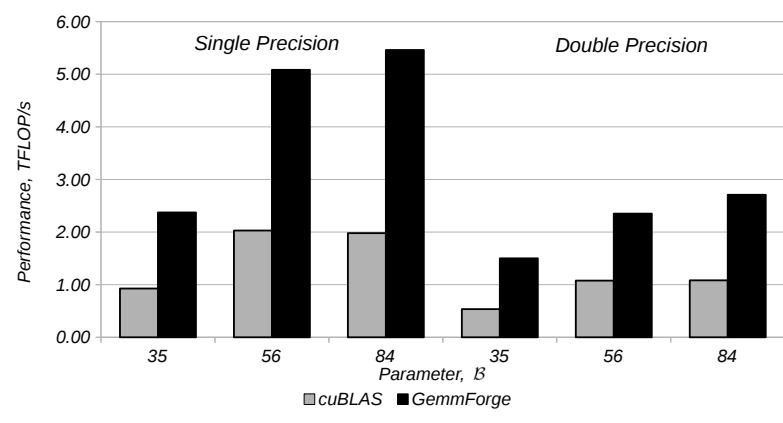

Figure 5: Performance of the parameterized benchmarks (14) implemented with GemmForge vs. cuBLAS.

In general, performance of the first kernel exceeds the roofline by $4.5 \%$ on average. This can be explained as a result of efficient hardware warp scheduling, which aims to overlap computations with data transfers, due to a sufficient number of resident threads running on the same SM. In case of the second kernel, performance is approximately $10 \%$ lower than predicted by the model.

\subsection{Comparison with cuBLAS}

We implemented benchmark (14) with both GemmForge and cuBLAS libraries, using the cublas XgemmStridedBatched subroutine of the latter as an efficient batched GEMM implementation. Comparisons are shown in Fig. 5 where results were obtained with similar parameters and setup as in the previous experiments.

The results show that the generated kernels outperform cuBLAS by 2.5 times on average. Nvidia Visual Profiler reveals that the code generation approach results in a better memory utilization ${ }^{4}$ in contrast to cuBLAS (see Fig. 6). Deviations from the ideal utilization of the generated kernels probably result from misalignment of matrices on 128 byte boundaries which was done on purpose to obtain more realistic results. Additionally, occupancy ${ }^{5}$ of the two generated kernels achieved approximately $90.5 \%$ and $72.8 \%$, respectively. In contrast, occupancy of cublasSgemmStridedBatched did not exceed $30 \%$. We can conclude from these numbers that in general generated kernels can better hide latency of memory access instructions. Moreover, the kernels generated with GemmForge executed less control-flow operations in contrast to the cuBLAS implementation. By and large it indicates that the code generation approach is well suited for DG-like computations in spite of the involved complexity.

\subsection{Performance Evaluation with SeisSol-Proxy}

SeisSol-Proxy is a set of benchmarks that contains the main computational kernels of SeisSol. The proxy implementation is based on a single time cluster which can be also viewed as time integration according to the global time stepping scheme. The proxy can run individual kernels as well as combinations of them to collect performance metrics. The main goal of the proxy is to reflect results of all applied optimization techniques performed at compile time.

\footnotetext{
${ }^{4}$ traffic to/from a memory subsystem, relative to its peak value.

${ }^{5}$ the ratio of active warps to the maximum number of warps supported on a particular SM model.
}

Fig. 7 shows results obtained with convergence orders 5, 6 and 7 and with 625000/312500 elements in case of using the single/double precision floating point format. We denote Ader as an implementation of time integration of a Taylor expansion done with the CK procedure i.e., (3) and (4). Local and Neighbor correspond to macro-kernels (5) and (6) respectively.

The performance differences observable between Figures 5 and 7 are mainly explained by the presence of the EQSPP optimization, described in Sec. 4, which reduces matrix sizes to avoid unnecessary computations. As mentioned there, it improves time-to-solution, however, it also reduces arithmetic intensities of the involved computations.

In order to understand how time clustering can affect potential GPU performance of the ADER-DG method, we performed a series of tests, shown in Fig. 8, where we gradually increased the number of elements per cluster, while keeping the same order of convergence (here: order 6). For comparison, we performed the same tests using Intel Xeon Gold 6148 CPU with 20 cores.

These tests reveal that unlike on CPUs, where performance reaches the plateau almost immediately, a GPU simulation requires at least 32768 elements per cluster to reach $\approx 90 \%$ of the maximum achievable performance. We assume that the performance drop can be primarily related to the overheads involved in launching GPU kernels, i.e, communication with the device driver, passing kernel arguments via the bus, etc. However, careful code instrumentation and profiling with a subsequent data analysis will be required to fully understand and quantify this problem. Nevertheless, the aforementioned value should be treated as a threshold for cluster sizes, below which GPU performance drops rapidly.

Unlike with global time stepping, where such constraints can be easily satisfied, it is quite common to have a few small time clusters, when applying the clustered LTS algorithm (see Fig. 1). Moreover, the first time cluster, which has the highest update rate, often has an extremely small size and may thus not only cause poor GPU hardware performance for the cluster, but also affect strong scaling of the entire LTS method.

\section{SIMULATIONS}

In this section, we evaluate the performance of our GPU version of SeisSol on the Marconi100 HPC cluster located at the CINECA supercomputing centre. Marconi100 contains 980 compute nodes connected with a Mellanox Infiniband EDR network. Each node is equipped with 2 Power9 CPUs where each CPU controls 2 Nvidia Volta V100 graphics cards via NVLink 2.0. We used OpenMPI (version 3.1.5) compiled with the UCX layer, which supports both CUDA-aware and GPUDirect technologies.

\subsection{Layer Over Half-space scenario}

The Layer Over Half-space (LOH.1) benchmark [5] aims to simulate elastic wave propagation caused by a single point source between two different adjacent regions. A relatively thin and flat layer located at the top represents a sedimental layer. This layer has a depth of $1 \mathrm{~km}$, a density of $2600 \mathrm{~kg} / \mathrm{m}^{3}$ and the following Lamé parameters: $\lambda=20.8 \mathrm{GPa}$ and $\mu=10.4 \mathrm{GPa}$. The second region is much deeper and occupies the main part of the computation domain. Material parameters applied to this region are $\rho=2700 \mathrm{~kg} / \mathrm{m}^{3}, \lambda=32.4 \mathrm{GPa}$ 


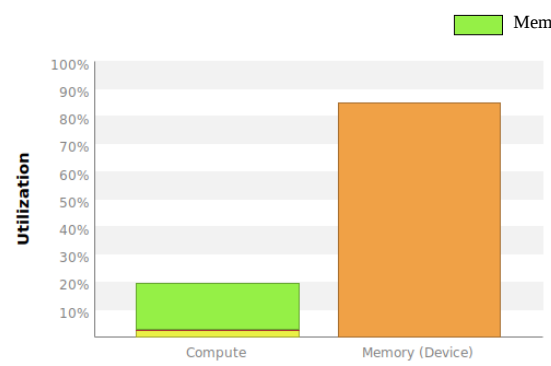

(a) 1st kernel: $T_{e}=A_{e} \cdot B_{e}$

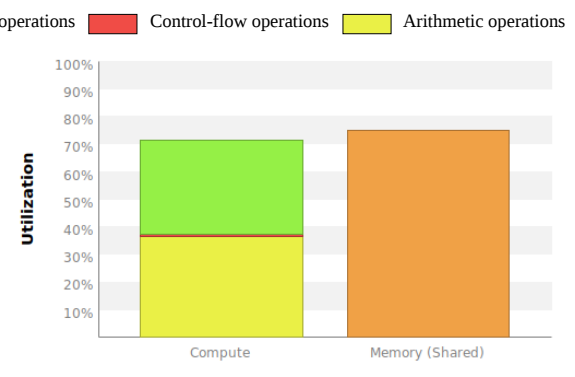

(b) 2nd kernel: $L_{e}=D \cdot T_{e}+L_{e}$.

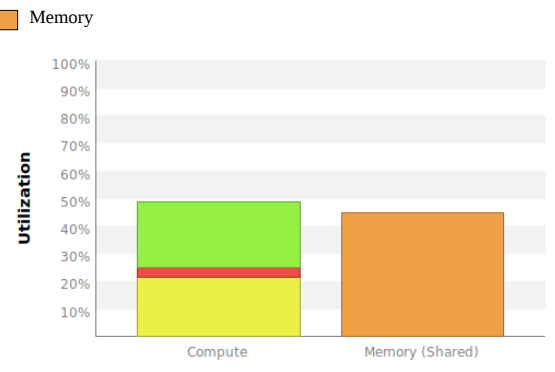

(c) cublasSgemmStridedBatched.

Figure 6: Compute utilization of kernels obtained with Nvidia Quadro P4000, single precision and $\mathcal{B}=56$.

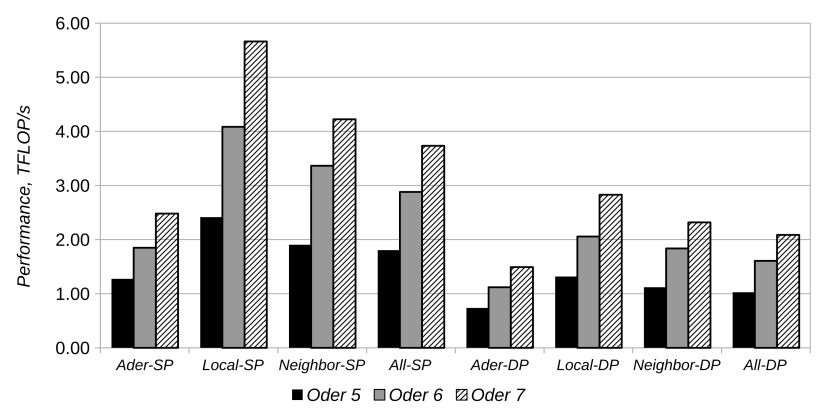

Figure 7: Performance of the main SeisSol compute-kernels obtained with the proxy application.

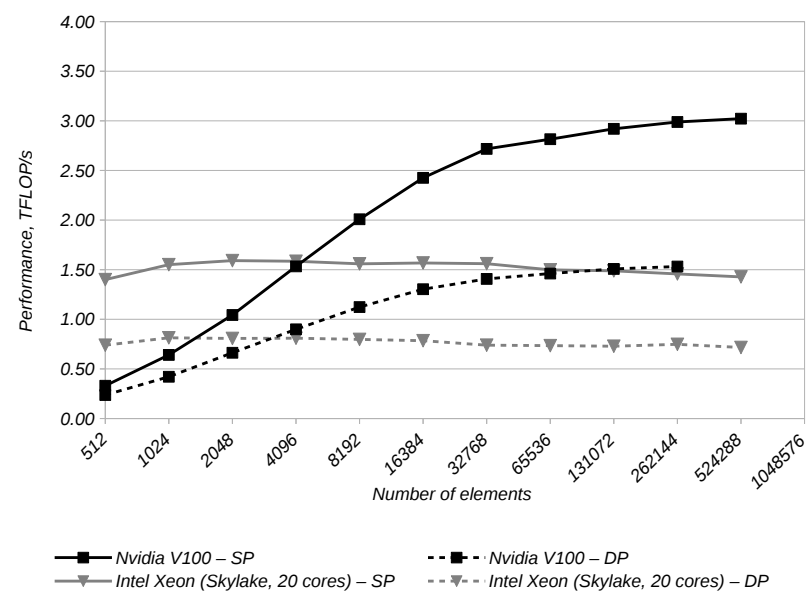

Figure 8: Influence of problem size on CPU and GPU performance.

and $\mu=32.4 \mathrm{GPa}$. Free surface conditions are applied at the top surface of the sedimental layer. All other domain boundaries are set to outflow boundary condition. The point source is located inside of the second region and at a total depth of $1.5 \mathrm{~km}$ shifted $4 \mathrm{~km}$ along both $x$ and $y$ axes from the origin.

The CAD model and mesh refinement regions of the benchmark were parametrized to generate meshes with different numbers of

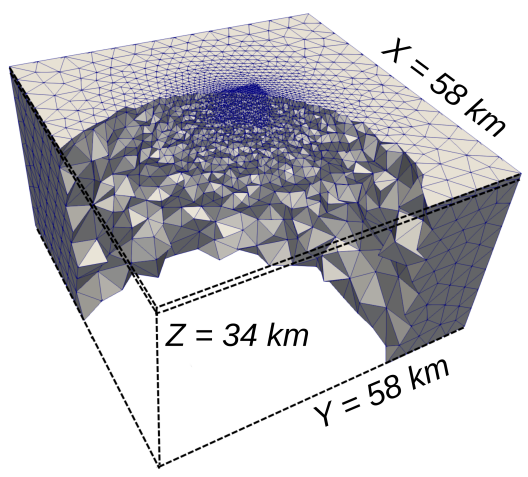

Figure 9: Example of a LOH.1 computational mesh.

elements but to preserve a similar distribution of small, medium and large cells as well as to keep the same size of the smallest elements and, as a result, a similar time integration step.

\subsection{Results}

7.2.1 Single GPU Performance. Fig. 10 shows the performance of the GPU version of SeisSol obtained using the LOH.1 benchmark with a single Nvidia V100 GPU. In this study, we compared performance of LTS and GTS schemes as well as influences of different convergence orders and floating point formats. Parameters of the CAD model were adjusted to generate approximately 625000 elements to allow comparison with the results obtained with the proxy application.

The simulation time was set to 1.5 seconds for each experiment which resulted in approximately 10000 time steps for the GTS scheme. We turned off writing results to files to exclude any influence from I/O operations.

Fig. 10 shows that performance of the GTS scheme is pretty close to the results obtained with the proxy application, whereas the LTS scheme turns out to be about $25 \%$ less efficient on average from a hardware utilization perspective. This reduced performance of the LTS scheme is expected and explained by the reduced performance for small time clusters, as detailed in Sec. 6.3.

For a review on the verification of the simulation results, see Appendix B. The results show that our GPU solver captures correct behavior of the elastic wave propagation model. Moreover, 


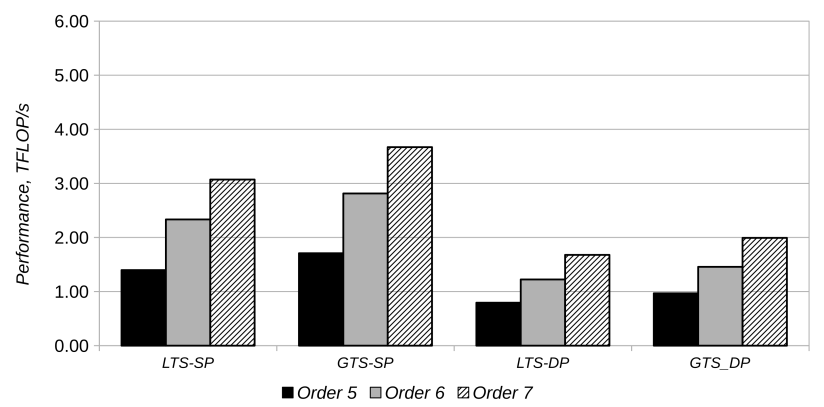

Figure 10: Comparisons of SeisSol performance using GTS and LTS schemes for the most commonly used convergence orders.

computing with the single precision floating point format does not introduce a significant numerical error for the LOH.1 scenario.

An analysis of tracing data obtained with Nvidia Visual Profiler revealed that evaluations of point sources, including time spent on transferring data between GPU and CPU and vise versa, took only $\approx 0.15 \%$ of time spent inside of the main computational loop. Therefore the overhead introduced by host-to-device communication of the GPU implementation of SeisSol is negligible for this particular scenario.

7.2.2 Multi-GPU Performance. In this part, we present a combined study of strong and weak scaling of the SeisSol GPU solver. Be aware that we cannot fulfill all requirements typically imposed to weak scaling tests: Because mesh generation and partitioning of unstructured meshes cannot be controlled perfectly, especially in case of the LTS scheme, we cannot precisely scale workloads between GPU instances while increasing the domain size. However, we can at least guarantee that the total number of elements increases proportionally relatively to the GPU count. For this set of experiments, we generated multiple computational meshes with numbers of elements in a range between 1.25 and 40 million.

The plots in Fig. 11 show results of strong and weak scaling obtained with both GTS and LTS schemes. The lines denoted as ideal correspond to ideal linear scaling of the corresponding first experiments, obtained with 4 GPUs (i.e., one node). The performance values are based on elapsed time, which also include overheads resulting from non-blocking MPI communication. As in the previous experiment, all I/O operations were turned off.

It can be seen from Fig. 11a and Fig. 11b, parallel efficiency of SeisSol with the GTS scheme reaches almost $93 \%$ in case of weak scaling, whereas, the worst efficiency among all strong scaling experiments is around $50 \%$. Such loss of strong scaling performance is caused by two distinct factors: an increase of MPI communication and a decrease of performance of each GPU instance caused by a reduced sub-domain size (cmp. Fig. 8).

Respective scaling experiments using the LTS scheme (Fig. 11c and Fig. 11d) result in mediocre hardware utilization. We observe significant deviations of performance metrics from their ideal lines. For example, a strong scaling experiment with a mesh containing 1.25 million elements showed that parallel efficiency dropped to approximately $72 \%$ for 64 GPUs and with double precision.
Despite of the lower performance values obtained with the LTS scheme, the scheme is always algorithmically more efficient than GTS. For the obtained results, local time stepping resulted in a reduction of time-to-solution by 1.3 times on average.

Several of the weak scaling experiments with the LTS method failed. We experienced significant imbalance in memory requirements between GPUs, which eventually led to a case when one or a few GPU instances ran out of memory. Fig. 12a shows an example of such a case where the 13th GPU instance got assigned 3 times more elements than the average value. However, at the same time the distribution of element-wise work loads, according to the definition given in (12), resulted in an imbalance of only $0.14 \%$. Hence, we face a mesh partitioning challenge, as we need to enforce additional constraints on the memory balancing. Conventional CPU systems usually come with a sufficient amount of on-board main memory, such mesh partitioning need not consider memory balancing. On GPU systems we are forced to use more GPU instances than optimally needed in order to just run a problem, which issue prevents us to optimally exploit hardware on GPU computing clusters. It indicates that we need to improve our mesh partitioning heuristic, for example by moving to multi-constraint graph partitioning. We also expect that modifying the clustering of elements in LTS, for example by merging smaller time clusters, should positively affect the overall execution time.

\section{CONCLUSION}

In this paper, we presented a version of SeisSol that simulates elastic wave propagation on distributed multi-GPU platforms. The scope of this work included a change in task decomposition, generating CUDA compute-kernels, and comparisons of global and local time stepping schemes from a hardware utilization perspective using strong and weak scaling analyses. There are several main outcomes from this research.

We developed a new open-source library, GemmForge, to generate GPU source code for small, fixed-size, dense batched matrix multiplications, which resulted in 2.5 average speed-up compared to the corresponding cuBLAS subroutines. We analysed the generated code using the Roofline model. We showed that the generated CUDA kernels performed well, being limited by the memory bandwidth due to low arithmetic intensities. We will address this issue in our follow-up research by considering a fusion of several matrix multiplication kernels.

We examined the influence of problem size on the performance of the ADER-DG method for both GPU and CPU implementations. While we achieved a factor of 2 speed-up relative to an Intel Xeon Gold 6148 CPU with 20 cores, we showed that GPU performance dropped rapidly below a certain problem size.

We analyzed the performance of SeisSol on the Marconi100 supcomputer, a distributed multi-GPU environment. We compared both the GTS and LTS scheme using weak and strong scaling scenarios. Results of scaling SeisSol with the LTS model have identified novel requirements on the GPU implementation of the LTS method and on the corresponding mesh partitioning. 


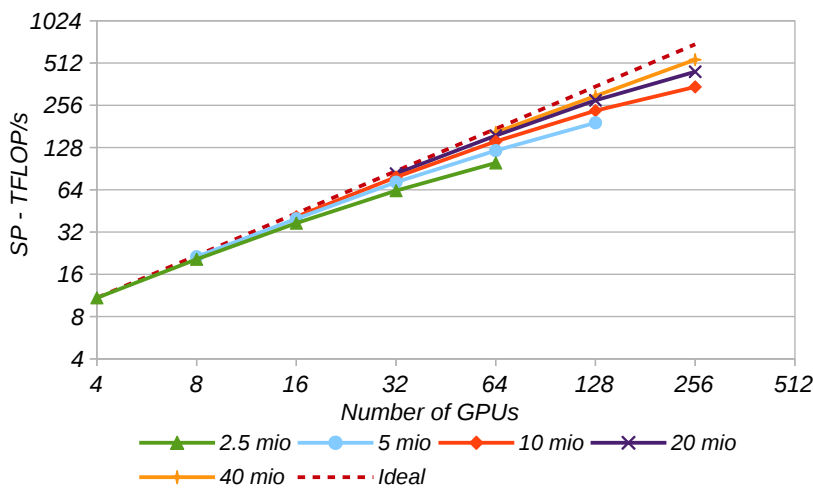

(a) GTS: Single Precision.

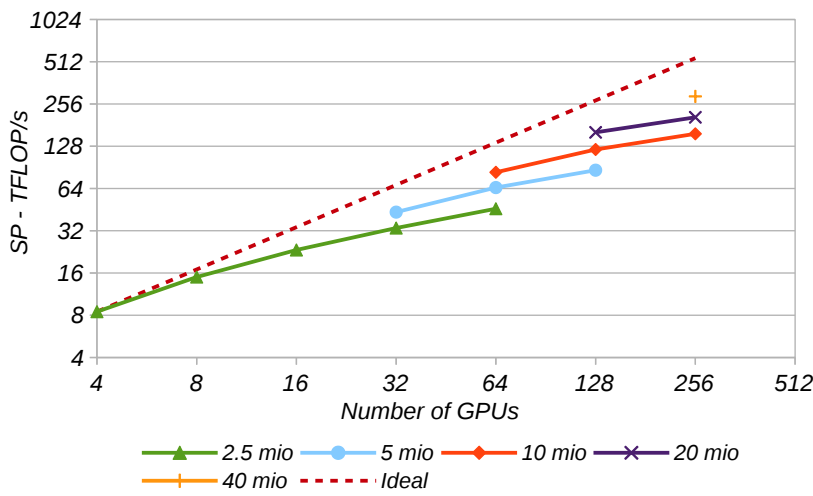

(c) LTS: Single Precision.

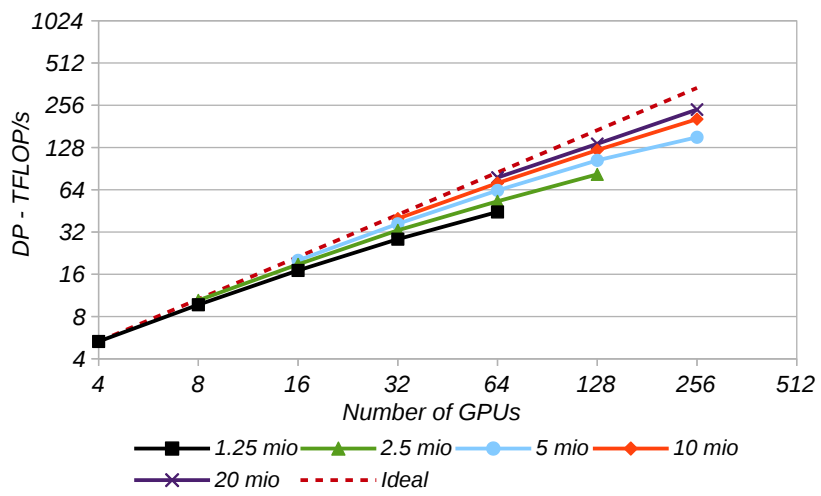

(b) GTS: Double Precision.

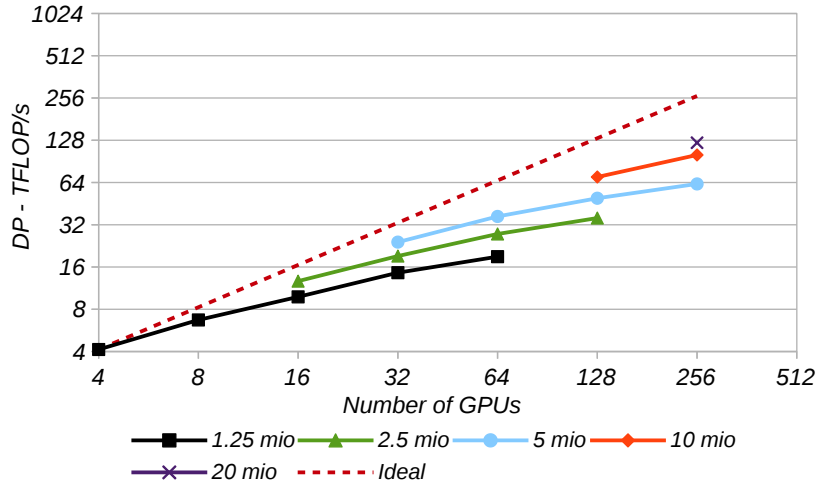

(d) LTS: Double Precision.

Figure 11: Strong/Weak scaling of LOH.1 benchmark obtained with the GTS/LTS schemes and convergence order 6.

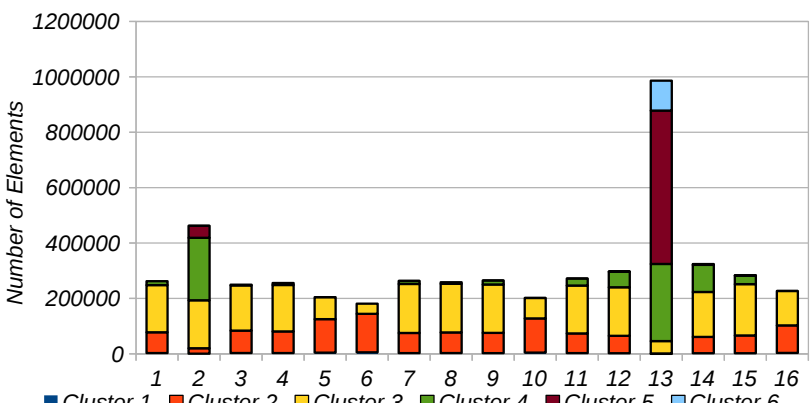

(a) Element distribution between MPI processes and LTS clusters.

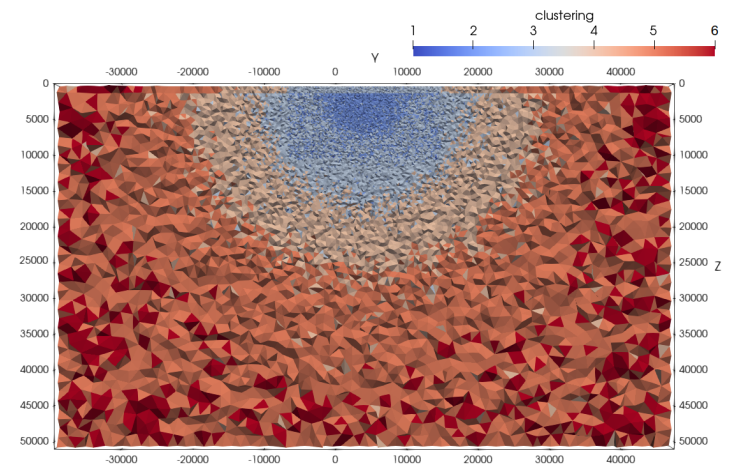

(b) Cross section of the mesh across $\mathbf{x}$ axis trough the origin, where each color represents a cluster

Figure 12: LTS clustering of 5 million elements over 6 time clusters between 16 partitions.

\section{ACKNOWLEDGMENTS}

The research leading to these results has received funding from the European Union's Horizon 2020 research and innovation programme under the ChEESE project, grant agreement No. 823844. We acknowledge PRACE for awarding us access to the Marconi100 supercomputer at the CINECA supercomputing centre, Italy. We especially thank all involved people at CINECA, in particular Piero Lanucara, for their support.

\section{REFERENCES}

[1] Daniel S Abdi, Lucas C Wilcox, Timothy C Warburton, and Francis X Giraldo. 2019. A GPU-accelerated continuous and discontinuous Galerkin non-hydrostatic atmospheric model. Int. J. High Perform. Comp. App. 33, 1 (2019), 81-109. 
[2] Alexander Breuer, Alexander Heinecke, and Michael Bader. 2016. Petascale local time stepping for the ADER-DG finite element method. In 2016 IEEE International Parallel and Distributed Processing Symposium (IPDPS). IEEE, 854-863.

[3] Alexander Breuer, Alexander Heinecke, and Yifeng Cui. 2017. EDGE: Extreme scale fused seismic simulations with the discontinuous Galerkin method. In International Supercomputing Conference, ISC'17. Springer, 41-60.

[4] Jieyang Chen, Nan Xiong, Xin Liang, Dingwen Tao, Sihuan Li, Kaiming Ouyang, Kai Zhao, Nathan DeBardeleben, Qiang Guan, and Zizhong Chen. 2019. TSM2: optimizing tall-and-skinny matrix-matrix multiplication on GPUs. In Proceedings of the ACM International Conference on Supercomputing. 106-116.

[5] Steven M Day, J Bielak, D Dreger, R Graves, S Larsen, KB Olsen, and A Pitarka. 2003. Tests of 3D elastodynamic codes: Final report for Lifelines Project $1 \mathrm{~A} 02$. Pacific Earthquake Engineering Research Center (2003).

[6] Edoardo Di Napoli, Diego Fabregat-Traver, Gregorio Quintana-Ortí, and Paolo Bientinesi. 2014. Towards an efficient use of the BLAS library for multilinear tensor contractions. Appl. Math. Comput. 235 (2014), 454-468.

[7] Michael Dumbser and Martin Käser. 2006. An arbitrary high-order discontinuous Galerkin method for elastic waves on unstructured meshes-II. The three-dimensional isotropic case. Geophys. 7. Int. 167, 1 (2006), 319-336.

[8] Michael Dumbser and Claus-Dieter Munz. 2006. Building blocks for arbitrary high order discontinuous Galerkin schemes. 7. Sci. Comput. 27, 1-3 (2006), 215-230.

[9] Dominik Ernst, Georg Hager, Jonas Thies, and Gerhard Wellein. 2020. Performance Engineering for a Tall \& Skinny Matrix Multiplication Kernels on GPUs. In Parallel Processing and Applied Mathematics. Springer, 505-515.

[10] Ruth A. Harris, Michael Barall, Brad Aagaard, Shuo Ma, Daniel Roten, Kim Olsen, Benchun Duan, Dunyu Liu, Bin Luo, Kangchen Bai, Jean-Paul Ampuero, Yoshihiro Kaneko, Alice-Agnes Gabriel, Kenneth Duru, Thomas Ulrich, Stephanie Wollherr, Zheqiang Shi, Eric Dunham, Sam Bydlon, Zhenguo Zhang, Xiaofei Chen, Surendra Nadh Somala, Christian Pelties, Josué Tago, Victor Manuel CruzAtienza, Jeremy Kozdon, Eric Daub, Khurram Aslam, Yuko Kase, Kyle Withers, and Luis Dalguer. 2018. A Suite of Exercises for Verifying Dynamic Earthquake Rupture Codes. Seismol.l Res. Lett. 89, 3 (04 2018), 1146-1162.

[11] Alexander Heinecke, Alexander Breuer, Sebastian Rettenberger, Michael Bader, Alice-Agnes Gabriel, Christian Pelties, Arndt Bode, William Barth, Xiang-Ke Liao, Karthikeyan Vaidyanathan, Mikhail Smelyanskiy, and Pradeep Dubey. 2014 Petascale High Order Dynamic Rupture Earthquake Simulations on Heterogeneous Supercomputers. In SC '14: Proceedings of the International Conference for High Performance Computing, Networking, Storage and Analysis. IEEE, 3-14.

[12] A. Heinecke, G. Henry, M. Hutchinson, and H. Pabst. 2016. LIBXSMM: Accelerating Small Matrix Multiplications by Runtime Code Generation. In SC16: Int. Conf. for HPC, Networking, Storage and Analysis. 981-991.

[13] Zhe Jia, Marco Maggioni, Benjamin Staiger, and Daniele Paolo Scarpazza. 2018 Dissecting the NVIDIA Volta GPU Architecture via Microbenchmarking. CoRR abs/1804.06826 (2018). arXiv preprint arXiv:1804.06826 (2018).

[14] George Karypis and Vipin Kumar. 2009. MeTis: Unstructured Graph Partitioning and Sparse Matrix Ordering System, Version 4.0. http://www.cs.umn.edu/ metis.

[15] Martin Käser, Michael Dumbser, Josep De La Puente, and Heiner Igel. 2007. An arbitrary high-order discontinuous Galerkin method for elastic waves on unstructured meshes-III. Viscoelastic attenuation. Geophys. F. Int. 168, 1 (2007), 224-242.

[16] Martin Käser, P Martin Mai, and Michael Dumbser. 2007. Accurate calculation of fault-rupture models using the high-order discontinuous Galerkin method on tetrahedral meshes. B. Seismol. Soc. Am. 97, 5 (2007), 1570-1586.

[17] Stefano Markidis, Steven Wei Der Chien, Erwin Laure, Ivy Bo Peng, and Jeffrey S Vetter. 2018. Nvidia tensor core programmability, performance \& precision. In 2018 IEEE International Parallel and Distributed Processing Symposium Workshops (IPDPSW). IEEE, 522-531.

[18] Axel Modave, Amik St-Cyr, and Tim Warburton. 2016. GPU performance analysis of a nodal discontinuous Galerkin method for acoustic and elastic models. Computers \& Geosciences 91 (2016), 64-76.

[19] Tesla NVIDIA. 2017. NVIDIA Tesla V100 GPU Architecture.

[20] Max Rietmann, Peter Messmer, Tarje Nissen-Meyer, Daniel Peter, Piero Basini, Dimitri Komatitsch, Olaf Schenk, Jeroen Tromp, Lapo Boschi, and Domenico Giardini. 2012. Forward and adjoint simulations of seismic wave propagation on emerging large-scale GPU architectures. In SC'12: Proceedings of the International Conference on High Performance Computing, Networking, Storage and Analysis. IEEE, $1-11$

[21] Max Rietmann, Daniel Peter, Olaf Schenk, Bora Uçar, and Marcus Grote. 2015 Load-balanced local time stepping for large-scale wave propagation. In 2015 IEEE International Parallel and Distributed Processing Symposium. IEEE, 925-935.

[22] Paul Springer and Paolo Bientinesi. 2018. Design of a high-performance gemm like tensor-tensor multiplication. ACM Trans. Math. Software 44, 3 (2018), 1-29.

[23] Kasia Świrydowicz, Noel Chalmers, Ali Karakus, and Tim Warburton. 2019. Acceleration of tensor-product operations for high-order finite element methods. Int. F. High Perform. Comp. App. 33, 4 (2019), 735-757.

[24] Thomas Ulrich, Alice-Agnes Gabriel, Jean-Paul Ampuero, and Wenbin Xu. 2019. Dynamic viability of the 2016 Mw 7.8 Kaikōura earthquake cascade on weak crustal faults. Nat. Commun. 10, 1 (2019), 1-16.

[25] Carsten Uphoff and Michael Bader. 2016. Generating high performance matrix kernels for earthquake simulations with viscoelastic attenuation. In 2016 International Conference on High Performance Computing \& Simulation (HPCS). IEEE, 908-916.

[26] Carsten Uphoff and Michael Bader. 2020. Yet Another Tensor Toolbox for discontinuous Galerkin methods and other applications. ACM Trans. Math. Software 46, 4 (2020). https://doi.org/10.1145/3406835

[27] Carsten Uphoff, Sebastian Rettenberger, Michael Bader, Elizabeth H. Madden, Thomas Ulrich, Stephanie Wollherr, and Alice-Agnes Gabriel. 2017. Extreme Scale Multi-Physics Simulations of the Tsunamigenic 2004 Sumatra Megathrust Earthquake. In SC '17: Proceedings of the International Conference for High Performance Computing, Networking, Storage and Analysis. ACM.

[28] Peter Vincent, Freddie Witherden, Brian Vermeire, Jin Seok Park, and Arvind Iyer. 2016. Towards green aviation with python at petascale. In SC'16: Proceedings of the International Conference for High Performance Computing, Networking, Storage and Analysis. IEEE, 1-11.

[29] Stephanie Wollherr, Alice-Agnes Gabriel, and P Martin Mai. 2019. Landers 1992 "reloaded": Integrative dynamic earthquake rupture modeling. 7. Geophys. Res.: Sol. Ea. 124, 7 (2019), 6666-6702. 


\section{A GEMMFORGE: GENERATED KERNELS A.1 CUDA Listing}

We provide an example of a batched GEMM kernel generated with GemmForge in order to provide a better view on the algorithm described in 5.5. For consistency with Section 6 we show the kernel of the second part of benchmark (15), namely: $L_{e}=D \cdot T_{e}+L_{e}$ where parameter $\mathcal{B}$ is equal to 56 . In this example, we use "strided" addressing mode, discussed in Section 5.5. Note that the code in Listing 1 is simplified to fit into the page size and augmented with comments. However, the main structural components are preserved. Ellipses shown within callSecondGemm are used to save space of the manuscript but, in fact, match the parameter list of the kernel.

\section{Listing 1: Generated batched GEMM kernel and launcher}

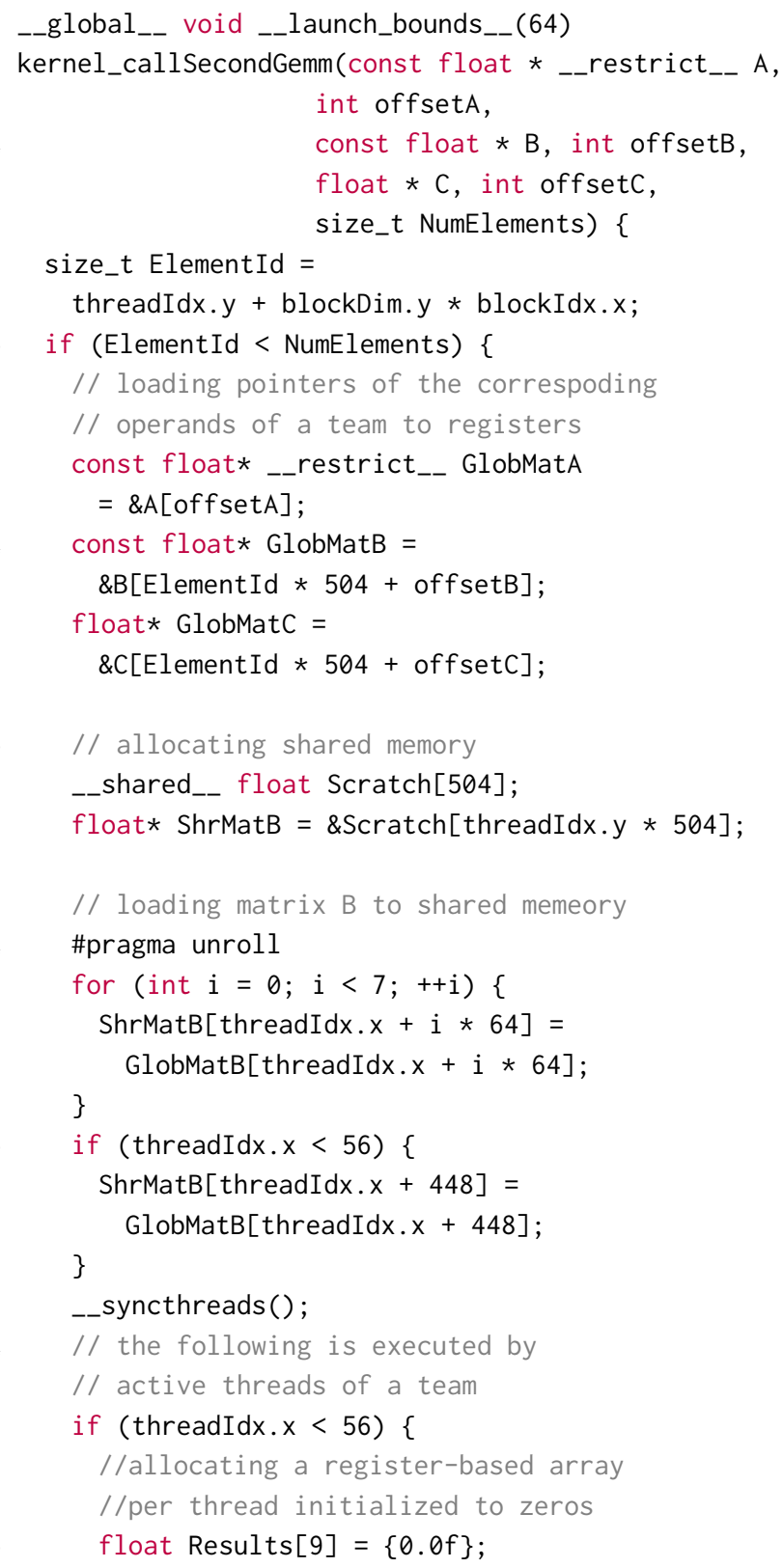

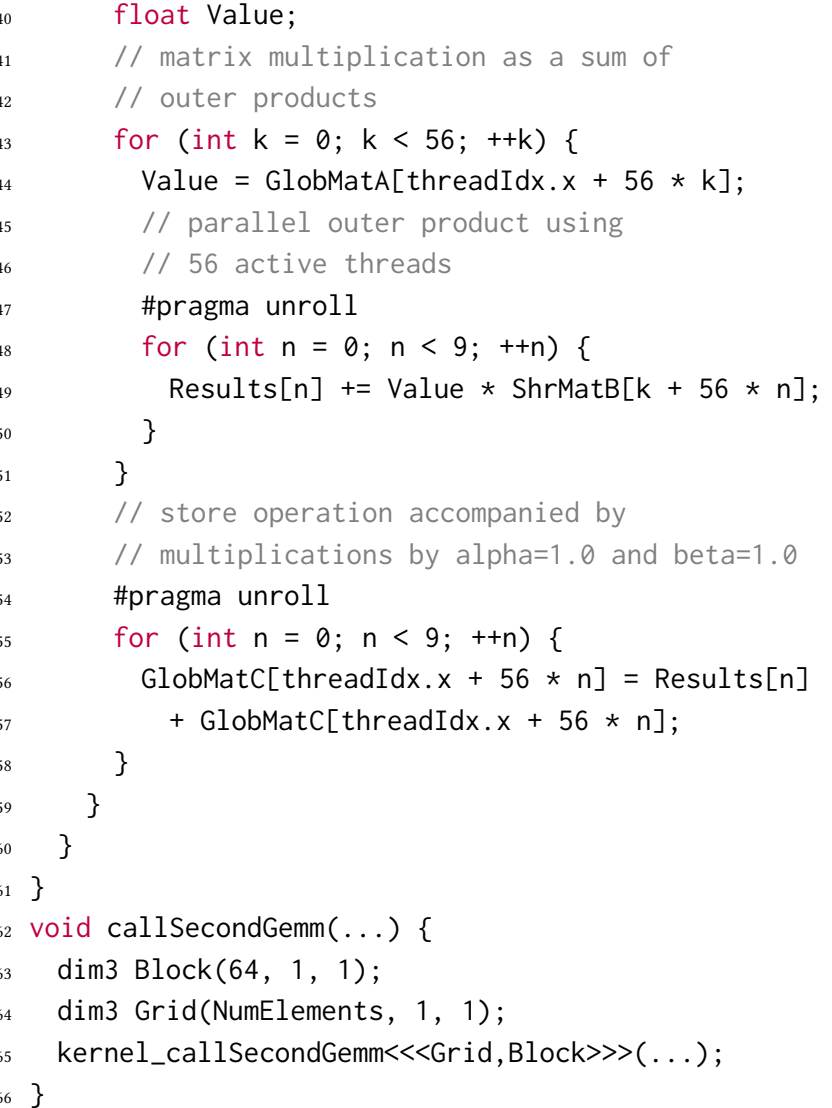

\section{A.2 Discussion}

The code shown in Listing 1 was generated by GemmForge for compute capability 7.0 and single precision floating point format.

GemmForge assists the $n v c c$ compiler by inserting hints, such as __restrict__ and __launch_bounds__, to achieve better register allocation and utilization in the resultant executable code. It is noteworthy that GemmForge can use different strategies to load operands residing in the global memory to shared memory. In this particular case, the library decides to use all threads of a team to load matrix $B$, because the leading dimension size of the matrix is smaller than the number of team threads. However, the library would load $B$ column-by-column if the difference between the leading dimension and the number of rows exceeded the number of team threads.

Having estimated run-time resources per matrix multiplication, GemmForge derives an optimal thread/block distribution based on information of the hardware limits of a target architecture. In this example, the library assigns only 64 threads per block to achieve 2048 resident threads per SM because a GPU with compute capability 7.0 can support up to 32 resident blocks per SM.

Offsets of matrices $A, B$ and $C$ shown in the parameter list of the kernel can be used for addressing element local matrices stored as arrays of structures. This becomes especially handy when a batch operand is provided as an array of pointers, because the same array can then be reused for multiple batched operations by applying necessary shifts. 


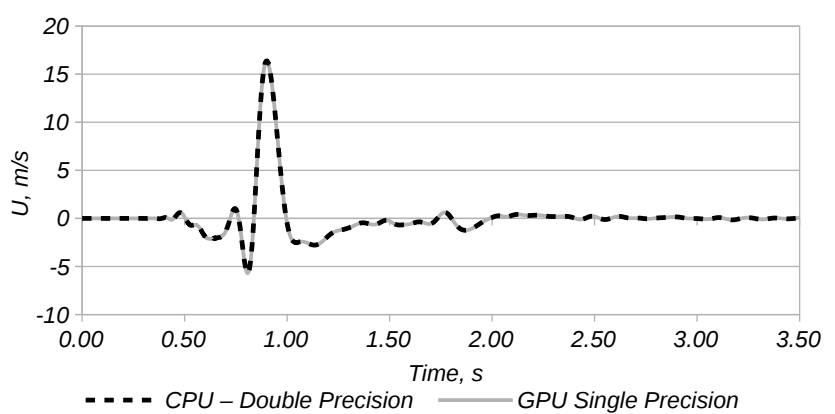

(a) Evolution of $U$ velocity component over time.

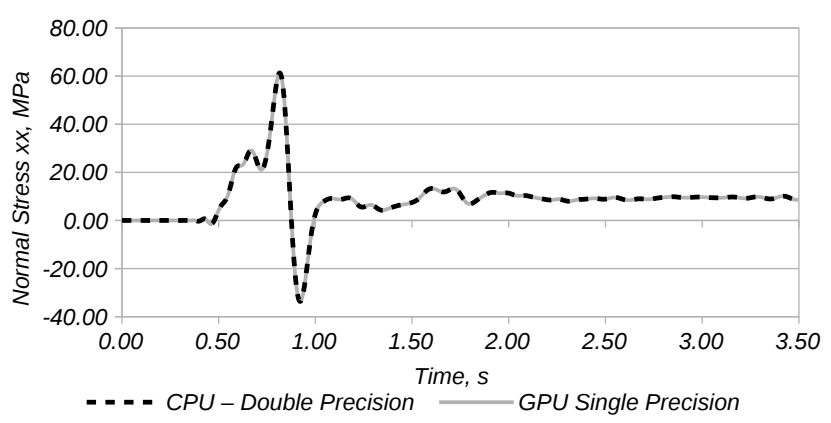

(b) Evolution of normal stress $x x$ over time.

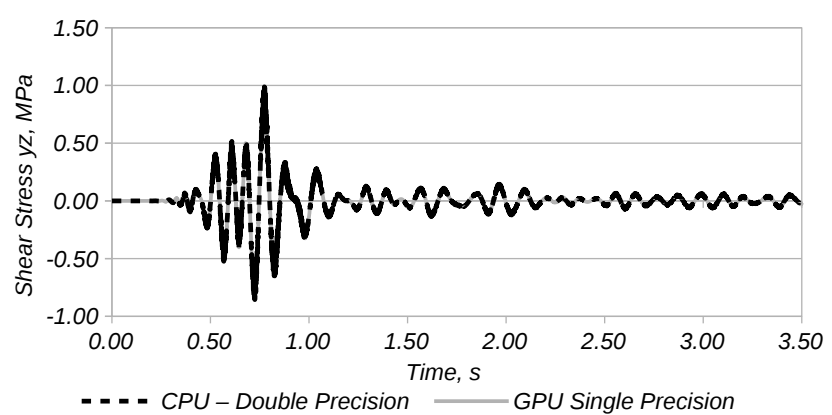

(c) Evolution of shear stress $y z$ over time.

Figure 13: Example plots for the evolution of one velocity (a) and two stress components $(b, c)$ in the LOH1 benchmark.

Table 2: Deviations of the GPU solver from the CPU one.

\begin{tabular}{|l|c|c|c|c|}
\hline Precision & \multicolumn{2}{|c|}{ Double } & \multicolumn{2}{c|}{ Single } \\
\hline Norm & $L_{2}$ & $L_{\infty}$ & $L_{2}$ & $L_{\infty}$ \\
\hline$u, m / s$ & $2.27 \mathrm{e}-17$ & $1.43 \mathrm{e}-18$ & $1.09 \mathrm{e}-08$ & $4.92 \mathrm{e}-10$ \\
\hline$v, m / s$ & $1.68 \mathrm{e}-17$ & $9.51 \mathrm{e}-19$ & $8.57 \mathrm{e}-09$ & $3.63 \mathrm{e}-10$ \\
\hline$w, m / s$ & $1.34 \mathrm{e}-17$ & $6.36 \mathrm{e}-19$ & $7.20 \mathrm{e}-09$ & $2.70 \mathrm{e}-10$ \\
\hline$\sigma_{x x}, M P a$ & $9.09 \mathrm{e}-11$ & $4.75 \mathrm{e}-12$ & $3.83 \mathrm{e}-02$ & $1.56 \mathrm{e}-03$ \\
\hline$\sigma_{y y}, M P a$ & $7.92 \mathrm{e}-11$ & $4.93 \mathrm{e}-12$ & $4.25 \mathrm{e}-02$ & $1.67 \mathrm{e}-03$ \\
\hline$\sigma_{z z}, M P a$ & $6.90 \mathrm{e}-12$ & $3.23 \mathrm{e}-13$ & $2.43 \mathrm{e}-03$ & $1.44 \mathrm{e}-04$ \\
\hline$\sigma_{x y}, M P a$ & $4.66 \mathrm{e}-11$ & $3.15 \mathrm{e}-12$ & $2.18 \mathrm{e}-02$ & $9.31 \mathrm{e}-04$ \\
\hline$\sigma_{y z}, M P a$ & $8.03 \mathrm{e}-12$ & $3.26 \mathrm{e}-13$ & $3.07 \mathrm{e}-03$ & $1.41 \mathrm{e}-04$ \\
\hline$\sigma_{x z}, M P a$ & $8.04 \mathrm{e}-12$ & $2.99 \mathrm{e}-13$ & $4.11 \mathrm{e}-03$ & $2.36 \mathrm{e}-04$ \\
\hline
\end{tabular}

\section{B VERIFICATION OF NUMERICAL RESULTS}

In the following we summarize verification of obtained results to guarantee the correctness of our GPU implementation. The CPU version of SeisSol is regularly verified against a suite of benchmarks [10] (see also the benchmark setups provided at https:// seissol.readthedocs.io/en/latest/cookbook_overview.html) and has been validated against various real events [24, 27, 29, e.g.]. We therefore use the CPU implementation as reference and compare physical quantities, caused by elastic wave propagation in a solid media, obtained with our GPU implementation. For consistency with the main manuscript we show results for the LOH.1 benchmark, described in Section 7.1, for arbitrary chosen points within a computational domain.

The verification shows that our GPU solver is correct and consistent with the reference implementation.

\section{B.1 Implementation}

To ensure correctness of computations obtained with our GPU solver, we used 20 points arbitrary placed within the computational domain of the LOH.1 benchmark. Values of the physical quantities were recorded with a time resolution of 0.5 milliseconds. The total simulation time was set to 3.5 seconds which resulted in 7000 records for each point. Due to a huge amount of collected data, we only show results obtained at a point located $\approx 3980 \mathrm{~km}$ away from the source with absolute coordinates equal to (1200.0, 1600.0, 0.1 ) from the origin - see the plots in Figure 13. Note that in this comparison we used double precision with our CPU reference implementation, but single precision for the GPU results.

Table 2 summarizes deviations of the numerical results generated with our GPU solver from the ones obtained with the reference implementation. The deviations are expressed via the $L_{2}$ and $L_{\infty}$ norms.

\section{B.2 Discussion and Summary}

As it can be seen, our GPU solver accurately captures correct behavior of the wave propagation process in case of both double and single precision. However, one can observe that the error increases for all stress components regardless of the floating point format. Nevertheless, this is still in an acceptable range. It is very likely that this occurs because SeisSol computes stress components in the Pascal $(\mathrm{Pa})$ physical units which results in handling large numerical values. 